\title{
Correction to: 3.5 ps burst mode pulses based on all-normal dispersion harmonic mode-locked
}

\author{
Haolin Yang ${ }^{1} \mathbb{D} \cdot$ Yue Chen ${ }^{1} \cdot$ Kaili Ding $^{1} \cdot$ Fuqiang Jia ${ }^{1} \cdot$ Kang $^{2}{ }^{2} \cdot$ Nigel Copner $^{2}$
}

Published online: 2 June 2021

○) Springer-Verlag GmbH Germany, part of Springer Nature 2021

Correction to: Applied Physics B (2020) 126:127

$$
\text { https://doi.org/10.1007/s00340-020-07481-w }
$$

Unfortunately, the corresponding author name was incorrectly published in the original publication. The complete correct name should read as follows

Fuqiang Jia

Publisher's Note Springer Nature remains neutral with regard to jurisdictional claims in published maps and institutional affiliations.

The original article can be found online at https://doi.org/10.1007/ s00340-020-07481-w.

Fuqiang Jia

jiafq@xmu.edu.cn

Haolin Yang

23120171153004@stu.xmu.edu.cn

Kang Li

kang.li@southwales.ac.uk

1 School of Electronic Science and Engineering, Xiamen University, Fujian 361005, Xiamen, China

2 Wireless and Optoelectronics Research and Innovation Centre, Faculty of Computing, Engineering and Science, University of South Wales, Wales, UK 